\title{
PT-symmetry entails pseudo-Hermiticity regardless of diagonalizability
}

\author{
Ruili Zhang, ${ }^{1}$ Hong Qin, ${ }^{2,3, \text {, }}$ and Jianyuan Xiao ${ }^{3}$ \\ ${ }^{1}$ School of Science, Beijing Jiaotong University, Beijing 100044, China \\ ${ }^{2}$ Plasma Physics Laboratory, Princeton University, Princeton, NewJersey 08543, USA \\ ${ }^{3}$ School of Physical Sciences, University of Science \\ and Technology of China, Hefei 230026, China
}

\begin{abstract}
We prove that in finite dimensions, a Parity-Time (PT)-symmetric Hamiltonian is necessarily pseudo-Hermitian regardless of whether it is diagonalizable or not. This result is different from Mostafazadeh's, which requires the Hamiltonian to be diagonalizable. PT-symmetry breaking often occurs at exceptional points where the Hamiltonian is not diagonalizable. Our result implies that PT-symmetry breaking is equivalent to the onset of instabilities of pseudo-Hermitian systems, which was systematically studied by Krein et al. in 1950s. In particular, we show that the mechanism of PT-symmetry breaking is the resonance between eigenmodes with different Krein signatures.
\end{abstract}

\footnotetext{
* Corresponding author, hongqin@princeton.edu
} 
In quantum physics, observables are assumed to be Hermitian operators. Bender and collaborators [1 3] proposed to relax this fundamental assumption and considered ParityTime (PT)-symmetric operators. The concept and techniques of PT-symmetry have been applied to many branches of physics [4-19]. Although PT-symmetry was first studied in infinite-dimensional systems, many of the current applications are in finite dimensions.

When discussing PT-symmetry, a related property, pseudo-Hermiticity, is often considered. Pseudo-Hermitian operators were introduced by Dirac and Pauli as a class of nonHermitian operators [20 22]. Investigating the relation between PT-symmetry and pseudoHermiticity may reveal important mathematical and physical structures of non-Hermitian operators. In this regard, Mostafazadeh proved that a diagonalizable PT-symmetric Hamiltonian is pseudo-Hermitian [23-25].

In this paper, we prove that in finite dimensions, a PT-symmetric Hamiltonian is necessarily pseudo-Hermitian regardless of whether it is diagonalizable or not. We first prove that for a Hamiltonian $H$, a sufficient and necessary condition of pseudo-Hermiticity is that $H$ is similar to its Hermitian conjugate $\bar{H}$ (Theorem 2). Then because a PT-symmetric Hamiltonian is similar to its Hermitian conjugate, it is pseudo-Hermitian (Theorem 3). We emphasize that this result is different from Mostafazadeh's [23 25]. The difference is significant, because our result relaxes the diagonalizability requirement. As we know, most of the interesting PT-symmetry breaking happens at exceptional points where the Hamiltonian is not diagonalizable. Our result is applicable when studying these effects.

As such an application, we show that a theoretical description of PT-symmetry breaking, which is arguably the most important topic in PT-symmetry physics, can be built upon the mathematical work on the instabilities of pseudo-Hermitian systems developed by Krein, Gel'fand and Lidskii [26-28] in 1950s. For a pseudo-Hermitian Hamiltonian, its eigenvalues are symmetric with respect to the real axis. As the system parameters vary, a necessary and sufficient condition for the onset of instability is that two eigenmodes with opposite Krein signatures collide, which is the so-called Krein collision. These results can be directly applied to PT-symmetric Hamiltonians due to Theorem 3 , implying that PT-symmetry breaking occurs when and only when eigenmodes with different Krein signatures collide. Note that when PT-symmetry breaking happens, the Hamiltonian can be either diagonalizable or non-diagonalizable. But PT-symmetry is often broken at the exceptional points where the Hamiltonian is not diagonalizable. As an example, we show that the governing equations 
of the classical Kelvin-Helmholtz instability, which was proven to be PT-symmetric [19], is pseudo-Hermitian, and the Kelvin-Helmholtz instability is the result of PT-symmetry breaking triggered by the Krein collision.

We start from the definitions of PT-symmetry, pseduo-Hermiticity, and another related concept, i.e., G-Hamiltonian matrix. Consider the linear system specified by a Hamiltonian $H$,

$$
\dot{\boldsymbol{x}}=-i H \boldsymbol{x}=A \boldsymbol{x},
$$

where $A$ is defined to be a shorthand notation of $-i H$.

The Hamiltonian $H$ in Eq. (1) is called PT-symmetric [1-3] if it commutes with the parity-time operator $P T$, i.e.,

$$
P T H-H P T=0 .
$$

Here $P$ is a linear operator satisfying $P^{2}=I$ and $T$ is the complex conjugate operator. In the present study, we will focus on finite-dimensional systems, for which $H, A$ and $P$ can be represented by matrices, and Eq. (2) is equivalent to

$$
P \bar{H}-H P=0
$$

where $\bar{H}$ denotes the complex conjugates of $H$.

The Hamiltonian $H$ in Eq. (1) is called pseudo-Hermitian [20 22] if there exits a nonsingular Hermitian matrix $G$ such that

$$
H^{\dagger} G-G H=0
$$

where $H^{\dagger}$ is the conjugate transpose of the matrix $H$.

The matrix $A=-i H$ in Eq. (1) is called G-Hamiltonian [26 28] if there exist a nonsingular Hermitian matrix $G$ and a Hermitian matrix $S$ such that

$$
A=i G^{-1} S
$$

The concept of pseudo-Hermiticity was first introduced by Dirac and Pauli in 1940s [2022]. G-Hamiltonian matrix was defined by Krein et al. in 1950s [26-28] in the study of linear dynamical systems satisfying the G-Hamiltonian condition (5). For finite-dimensional systems, these two concepts are equivalent. 
Theorem 1. For a finite-dimensional system $\dot{\boldsymbol{x}}=-i H \boldsymbol{x}=A \boldsymbol{x}, H$ is pseudo-Hermitian if and only if $A$ is a G-Hamiltonian matrix.

The proof of Theorem 1 is straightforward according to the definitions of pseudoHermitian and G-Hamiltonian matrices. But we give this fact the status of a theorem to highlight the exact equivalence between these two concepts independently defined by physicists and mathematicians. We will mostly use the terminology of pseudo-Hermiticity exclusively hereafter.

Now we establish a necessary and sufficient condition for pseudo-Hermiticity.

Theorem 2. For a matrix $H \in C^{n \times n}$, it is pseudo-Hermitian if and if only it is similar to its complex conjugate $\bar{H}$.

Proof. Necessity is easy to prove. If a Hamiltonian is pseudo-Hermitian, i.e., satisfying Eq. (4), then $H=G^{-1} H^{\dagger} G$. Thus matrix $H$ is similar to $H^{\dagger}$, and also to $\bar{H}$.

We prove the sufficiency by constructing the Hermitian matrix $G$. Matrix $H$ can be written as

$$
H=Q^{-1} J Q
$$

where $J$ is its Jordan canonical form and $Q$ is a reversible matrix. The Jordan canonical form consists of several Jordan blocks of the form

$$
J(\lambda)=\left(\begin{array}{cccc}
\lambda & 1 & & \\
& \ddots & \ddots & \\
& & \lambda & 1 \\
& & & \lambda
\end{array}\right)_{m \times m} .
$$

When $m=1$, the Jordan block $J(\lambda)$ is reduced to $\lambda$. If $H$ is similar to $\bar{H}$, then its eigenvalues are symmetric with respect to the real axis, and they are either real numbers or complex number pairs of the form $\lambda=a+b i$ and $\bar{\lambda}=a-b i$, where $a$ and $b$ are real numbers. Accordingly, there are two kinds of matrix blocks

$$
F_{1}=J(a)=\left(\begin{array}{cccc}
a & 1 & & \\
& \ddots & \ddots & \\
& & a & 1 \\
& & & a
\end{array}\right)_{m \times m} \text { and } F_{2}=\left(\begin{array}{cc}
J(a+b i) & 0 \\
0 & J(a-b i)
\end{array}\right)_{2 l \times 2 l}
$$


The Jordan matrix can now be expressed as $J=\operatorname{Diag}\left(M_{1}, M_{2}, \cdots, M_{k}\right)$, where $M_{j}$ is in the form of $F_{1}$ or $F_{2}$. In the following, we prove that both types of matrix blocks are pseudo-Hermitian. For both types of matrix blocks, we find that Hermitian matrix

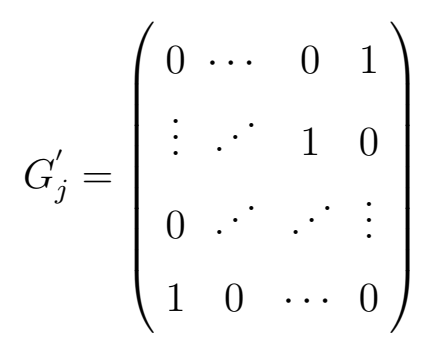

satisfies the condition $M_{j}^{\dagger} G_{j}^{\prime}-G_{j}^{\prime} M_{j}=0$, i.e., $M_{j}$ is pseudo-Hermitian. Next we construct a larger Hermitian matrix $G^{\prime}$ using $G_{j}^{\prime}$ as follows,

$$
G^{\prime}=\operatorname{Diag}\left(G_{1}^{\prime}, G_{2}^{\prime}, \cdots, G_{k}^{\prime}\right),
$$

and the Jordan canonical form of $H$ satisfies $J^{\dagger} G^{\prime}-G^{\prime} J=0$. Let

$$
G=Q^{\dagger} G^{\prime} Q
$$

and we obtain

$$
\begin{aligned}
H^{\dagger} G-G H & =\left(Q^{-1} J Q\right)^{\dagger} G-G Q^{-1} J Q \\
& =Q^{\dagger} J^{\dagger} Q^{-\dagger} Q^{\dagger} G^{\prime} Q-Q^{\dagger} G^{\prime} Q Q^{-1} J Q \\
& =Q^{\dagger}\left(J^{\dagger} G^{\prime}-G^{\prime} J\right) Q \\
& =0,
\end{aligned}
$$

where $G$ is a non-singular Hermitian matrix. This completes the proof that $H$ is pseudoHermitian.

The theorem is proved by constructing a non-singular Hermitian matrix $G$ for the similarity transformation between $H$ and $\bar{H}$. But $G$ is not unique. For a given $H$, we can find more than one non-singular Hermitian matrices $G$. In practice, one does not need to follow the construction procedure given in Theorem 2 to find $G$. It is often found by direct calculation.

Theorem 3. For finite-dimensional systems, a PT-symmetric Hamiltonian H is necessarily pseudo-Hemitian.

Proof. By the definition of PT-symmetry, i.e., Eq. (3), $H$ is similar to $\bar{H}$. Thus, according to Theorem 2 , it is pseudo-Hermitian. 
Theorem 3 is the main theorem in this paper, and we would like to emphasize again that it holds regardless of whether $H$ is diagonalizable or not. We note that Mostafazadeh's result [23-25], which states that diagonalizable PT-symmetric Hamiltonians are pseudo-Hermitian, is different from Theorem 3 .

As an application of Theorem 3, we investigate the mechanism of PT-symmetry breaking in the framework of pseudo-Hermiticity. Theorem 3 implies that PT-breaking is equivalent to the onset of instabilities of pseudo-Hermitian matrices, which was systematically studied by Krein, Gel'fand and Lidskii [26-28] in 1950s. Specifically, the instability analysis of pseudoHermitian matrices gives a comprehensive description on how real eigenvalues of $H$ evolve into conjugate pairs of complex eigenvalues as the system parameters vary. Here we briefly summarize the main results. (i) The eigenvalues of a pseudo-Hermitian Hamiltonian $H$ are symmetric with respect to real axis. They are either real numbers or complex conjugate pairs. (ii) Let $\psi$ be an eigenmode (or eigenvector) of $H$, Krein product of $\psi$ can be defined as $[26-28]$

$$
\langle\psi, \psi\rangle=\psi^{\dagger} G \psi
$$

The sign of the Krein product is called Krein signature. It was found that the physical meaning of the Krein product is action [29], which is partially indicated by the fact that its dimension is [energy] $\times[$ time]. We will also refer to the Krein product as action, especially in the context of physics. (ii) The eigenvalues of $H$ can be classified according to the Krein products of the corresponding eigenvectors. An $r$-fold real eigenvalue $\lambda$ of $H$ with its eigensubspace $V_{\lambda}$ is called the first kind if all eigenmodes of $\lambda$ have positive actions, i.e., $\langle\boldsymbol{y}, \boldsymbol{y}\rangle>0$ for any $\boldsymbol{y} \neq 0$ in $V_{\lambda}$. It is called the second kind if all eigenmodes of $\lambda$ have negative actions. If there exists a zero-action eigenmode, then $\lambda$ is called an eigenvalue of mixed kind [28]. If an eigenvalue is the first kind or the second kind, it's called definite. (iii) The number of each kind of eigenvalues is determined by the Hermitian matrix $G$. Let $p$ be the number of positive eigenvalues and $q$ be the number of negative eigenvalues of the matrix $G$, then any pseudo-Hermitan Hamiltonian has $p$ eigenvalues of first kind and $q$ eigenvalues of second kind (counting multiplicity). (iv) The finite-dimensional pseudo-Hermitian Hamiltonian is strongly stable if and only if all of its eigenvalues lie on the real axis and are definite. Here, a pseudo-Hermitian Hamiltonian is strongly stable means that eigenvalues of all pseudoHermitian Hamiltonians in an open neighborhood of the parameter space lie on the real axis. As a result, a pseudo-Hermitian Hamiltonian becomes unstable when and only when 
a positive-action mode resonates with a negative-action mode. This is a process known as the Krein collision.

Applying these results to PT-symmetric Hamiltonians, we see that PT-symmetry breaking can happen only when a repeated eigenvalue appears as a result of two eigenmodes resonate. However, if two eigenmodes with the same sign of action resonate, then there is no PT-symmetry breaking. PT-symmetry breaking is triggered only when a positive-action mode resonates with a negative-action mode.

Let's look at an example. The governing equations for the classical Kelvin-Helmholtz instability in fluid dynamics was shown to be a complex system with the following PTsymmetric Hamiltonian [19]

$$
H=\left(\begin{array}{cc}
\frac{-k\left(-u_{10} \rho_{10}-2 u_{20} \rho_{20}+u_{10} \rho_{20}\right)}{\rho_{10}+\rho_{20}} & \frac{-i|k|\left(u_{10}-u_{20}\right)^{2} \rho_{20}+i g\left(\rho_{20}-\rho_{10}\right)}{\rho_{10}+\rho_{20}} \\
-i|k| & k u_{10}
\end{array}\right)
$$

According to Theorem 3, it is also a pseudo-Hermitian Hamiltonian satisfying Eq. (4). With straightforward calculation, we find the following Hermitian matrix

$$
G=\left(\begin{array}{cc}
-|k| & 0 \\
0 & \frac{|k|\left(u_{10}-u_{20}\right)^{2} \rho_{20}-g\left(\rho_{20}-\rho_{10}\right)}{\rho_{10}+\rho_{20}}
\end{array}\right)
$$

such that $H^{\dagger} G-G H=0$. The eigenvalues of $H$ are

$$
\begin{aligned}
& a_{1}=\frac{k\left(\rho_{10} u_{10}+\rho_{20} u_{20}\right)-\sqrt{\Delta}}{\rho_{10}+\rho_{20}}, \\
& a_{2}=\frac{k\left(\rho_{10} u_{10}+\rho_{20} u_{20}\right)+\sqrt{\Delta}}{\rho_{10}+\rho_{20}}
\end{aligned}
$$

and the corresponding eigenvectors are

$$
\begin{aligned}
& \phi_{1}=\left(\frac{-i k \rho_{20}\left(u_{10}-u_{20}\right)+\sqrt{\Delta}}{|k|\left(\rho_{10}+\rho_{20}\right)}, 1\right), \\
& \phi_{2}=\left(\frac{-i k \rho_{20}\left(u_{10}-u_{20}\right)-\sqrt{\Delta}}{|k|\left(\rho_{10}+\rho_{20}\right)}, 1\right),
\end{aligned}
$$

where $\Delta=-|k| g\left(\rho_{10}^{2}-\rho_{20}^{2}\right)-k^{2} \rho_{10} \rho_{20}\left(u_{10}-u_{20}\right)^{2}$. The Krein signatures, or the signs of actions, of the eigenvalues of $H$ can be determined by the Hermitian matrix $G$. When

$$
\tau \equiv \frac{|k|\left(u_{10}-u_{20}\right)^{2} \rho_{20}-g\left(\rho_{20}-\rho_{10}\right)}{\rho_{10}+\rho_{20}}<0,
$$


(a) $u_{20}=2.3$

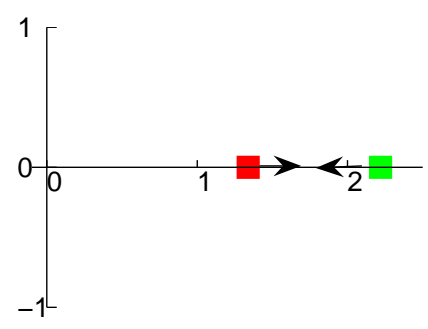

(c) $u_{20}=2.58114$

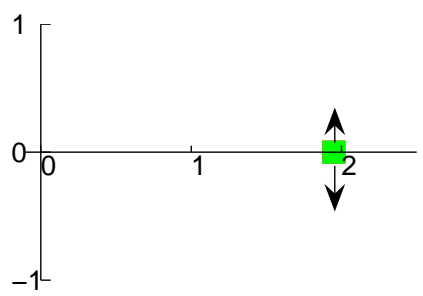

(b) $\mathrm{u}_{20}=2.5$

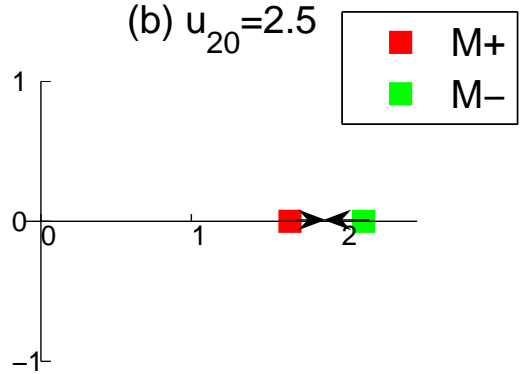

(d) $u_{20}=2.7$

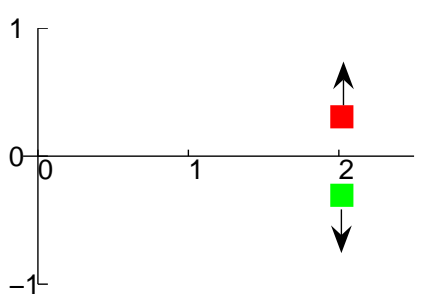

FIG. 1. PT-symmetry breaking occurs when a positive-action eigenmode (red) resonates with a negative-action eigenmode (green) .

both eigenvalues of $G$ are negative and the PT-symmetric Hamiltonian $H=i A$ is stable. When $\tau>0$, one of the eigenvalues of $G$ is positive and the other one is negative. Thus one eigenvalue of $H$ have a positive action and the other one has a negative action, and the resonance between them will result in PT-symmetry breaking. Let's use a numerically calculated examples to observe the breaking of PT-symmetry. We plot the process in Fig.1 by fixing $u_{10}=1, \rho_{10}=2, \rho_{20}=3, k=1$ and $g=3$, and varying $u_{20}$ from 2.3 to 2.7 . When $u_{20}=2.3$, the eigenvalues of $H$ are all real numbers, one of which has a positive action (marked by $M_{+}$) and the other one has a negative action (marked by $M_{-}$) in Fig.1(a). Fig.1. (b) shows that as $u_{20}$ increases, $M_{+}$and $M_{-}$move towards each other. Increasing $u_{20}$ to $\sqrt{5 / 2}+1=2.58114$, eigenmodes $M_{+}$and $M_{-}$collide on the real axis, as shown in Fig. 11(c). Because the resonance is between modes with different sign of actions, the eigenvalues of $H$ split into a pair symmetric with respect to the real axis and the PT-symmetry is broken. Fig.11(d) shows that the two eigenvalues of $H$ move out of real axis when $u_{20}=2.7$.

In summary, we have proved that for finite-dimensional systems, a PT-symmetric Hamiltonian is necessarily pseudo-Hermitian regardless of whether it is diagonalizable or not. This result is stronger than Mostafazadeh's [23 25], which requires that the Hamiltonian is diag- 
onalizable. As we know, PT-symmetry breaking often happens at exceptional points where the Hamiltonian is not diagonalizable. The fact that a PT-symmetric Hamiltonian is always pseudo-Hermitian implies that PT-symmetry breaking is equivalent to to the onset of instabilities of pseudo-Hermitian matrices. Therefore, the systematic results by Krein et al. on how a pseudo-Hermitian system becomes unstable [26 28] can be directly applied to the process of PT-symmetry breaking. In particular, we showed that PT-symmetry breaking is triggered when and only when two eigenmodes with different signs of actions resonate. This process is illustrated using the example of the classical Kelvin-Helmholtz instability.

We finish our discussion with an observation. Theorem 3 asserts that a PT-symmetric matrix is necessarily pseudo-Hermitian. One wonders whether the reverse is true. If the $P$ operator in the definition of PT-symmetry (2) is not required to be a parity transformation, i.e., $P^{2}=I$, then a pseudo-Hermitian matrix is also PT-symmetric according to Theorem 2 , In this case, PT-symmetry and pseudo-Hermition are equivalent, at least in finite dimensions. We note that essentially all the spectrum properties associated with PT-symmetry are still valid when the requirement of $P^{2}=I$ is removed.

\section{ACKNOWLEDGMENTS}

This research was supported by the National Natural Science Foundation of China (NSFC-11775219 and NSFC-11575186), the Fundamental Research Funds for the Central Universities (Grant No. 2017RC033), China Postdoctoral Science Foundation (2017LH002), the National Key Research and Development Program (2016YFA0400600, 2016YFA0400601, 2016YFA0400602 and 2017YFE0301700), and the U.S. Department of Energy (DE-AC0209CH11466).

[1] C. M. Bender and S. Boettcher, Phys. Rev. Lett. 80, 5243 (1998).

[2] C. M. Bender, D. C. Brody, and H. F. Jones, Phys. Rev. Lett. 89, 270401 (2002).

[3] C. M. Bender, Rep. Prog. Phys. 70, 947 (2007).

[4] H. Jones, Phys. Lett. A 262, 242 (1999).

[5] P. Dorey, C. Dunning, and R. Tateo, J. Phys. A: Math. Theor. 40, R205 (2007). 
[6] K. G. Makris, R. El-Ganainy, D. Christodoulides, and Z. H. Musslimani, Phys. Rev. Lett. 100, 103904 (2008).

[7] S. Klaiman, U. Günther, and N. Moiseyev, Phys. Rev. Lett. 101, 080402 (2008).

[8] S. Longhi, Phys. Rev. Lett. 103, 123601 (2009).

[9] H. Schomerus, Phys. Rev. Lett. 104, 233601 (2010).

[10] Y. Chong, L. Ge, and A. D. Stone, Phys. Rev. Lett. 106, 093902 (2011).

[11] L. Feng, M. Ayache, J. Huang, Y.-L. Xu, M.-H. Lu, Y.-F. Chen, Y. Fainman, and A. Scherer, Science 333, 729 (2011).

[12] A. Szameit, M. C. Rechtsman, O. Bahat-Treidel, and M. Segev, Phys. Rev. A 84, 021806 (2011).

[13] J. Schindler, A. Li, M. C. Zheng, F. M. Ellis, and T. Kottos, Phys. Rev. A 84, 040101 (2011).

[14] A. Regensburger, C. Bersch, M.-A. Miri, G. Onishchukov, D. N. Christodoulides, and U. Peschel, Nature 488, 167 (2012).

[15] B. Peng, Ş. K. Özdemir, F. Lei, F. Monifi, M. Gianfreda, G. L. Long, S. Fan, F. Nori, C. M. Bender, and L. Yang, Nat. Phys. 10, 394 (2014).

[16] M. J. Ablowitz and Z. H. Musslimani, Nonlinearity 29, 915 (2016).

[17] A. K. Jahromi, A. U. Hassan, D. N. Christodoulides, and A. F. Abouraddy, Nat. Commun. 8, 1359 (2017).

[18] H. Hodaei, A. U. Hassan, S. Wittek, H. Garcia-Gracia, R. El-Ganainy, D. N. Christodoulides, and M. Khajavikhan, Nature 548, 187 (2017).

[19] H. Qin, R. Zhang, A. S. Glasser, and J. Xiao, Phys. Plasmas 26, 032102 (2019).

[20] P. A. Driac, Proceedings of the Royal Society of London. Series A. Mathematical and Physical Sciences 180, 1 (1942).

[21] W. Pauli, Rev. Mod. Phys. 15, 175 (1943).

[22] T. D. Lee and G. C. Wich, Nucl. Phys. B 9, 209 (1969).

[23] A. Mostafazadeh, J. Math. Phys. 43, 205 (2002).

[24] A. Mostafazadeh, J. Math. Phys. 43, 2814 (2002).

[25] A. Mostafazadeh, J. Math. Phys. 43, 3944 (2002).

[26] M. Krein, Doklady Akad. Nauk. SSSR N.S. 73, 445 (1950).

[27] I. M. Gel'fand and V. B. Lidskii, Uspekhi Mat. Nauk 10, 3 (1955).

[28] V. Yakubovich and V. Starzhinskii, Linear Differential Equations with Periodic Coefficients, 
Vol. I (Wiley, 1975).

[29] R. Zhang, H. Qin, R. C. Davidson, J. Liu, and J. Xiao, Phys. Plasmas 23, 072111 (2016). 